\title{
WISSEN VERSTEHEN. NELSON GOODMANS SYMBOLTHEORETISCHE REVISION DES WISSENSBEGRIFFS
}

Wissen wird traditionell mit einer stetig anwachsenden Ansammlung wahrer Aussagen gleichgesetzt, die im Gegensatz zu Glauben und Meinen als unzweifelbar und gewiß gelten. Das Verständnis von Wissen, wie es bis heute die Diskussion prägt, wird noch immer von seinen aristotelischen Wurzeln her bestimmt. Wissen hatte jener als wahre Aussagen definiert, deren Richtigkeit die Überprüfung mit der Wirklichkeit beweist. Als Folge des menschlichen Strebens nach Wissen nimmt das, was gewußt wird, fortwährend zu.

Vorstellungen dieser Art bestimmen den gängigen Wissensbegriff. Demnach läßt sich Wissen in Aussagen fassen, als bereinigtes Endergebnis, losgelöst vom Prozeß der Gewinnung, von Forschung und Entdeckung. Wissen wächst stetig an: Durch die nicht endende Anhäufung von Neuem führt uns der ununterbrochene Zugewinn an Erkenntnis immer näher an ein absolutes Wissen heran. Wissen ist objektiv, es läßt sich unabhängig von subjektiven Verstehensprozessen nachweisen. Wissen ist wahr und unerschütterlich, was sich einmal als unzweifelbar und gewiß herausgestellt hat, gilt für alle vergangene, gegenwärtige und zukünftige Zeit. Was aber wahr ist, zeigt uns der Blick auf die Wirklichkeit. Bei Hegel kulminiert in gewisser Weise diese Ansicht: der Weg des Denkens mündet in das wahre oder »absolute « Wissen. Wissen und Wahrheit werden auf der letzten Stufe des Bewußtseins zur Einheit. Über das Verhältnis von Wissen und Wahrheit hinaus ist die Beziehung von Wissen und Glauben, die Hegel in der Jenaer Schrift von 1802 untersucht, von großer Wichtigkeit. ${ }^{1}$

Knapp zweihundert Jahre später hat sich die Lage radikal verändert. Viele der Voraussetzungen scheinen in der Philosophie Ende des 20. Jahrhunderts nicht mehr haltbar. Der amerikanische Symboltheoretiker Nelson Goodman unterzieht in seinem Spätwerk Ways of Worldmaking (1978) und Reconceptions in Philosophy and Other Arts and Sciences (1988, geschrieben zusammen mit Catherine Elgin) diese zentralen Begriffe der neuzeitlichen Erkenntnistheorie einer grundsätzlichen Neukonzeption. Wissen und Wahrheit werden zu Verstehen und Richtigkeit. Glaube jedoch tritt allenfalls noch in der Form von Gewißheit auf. Doch auch diese gilt es zu ersetzen: durch den wesentlich schwächeren Begriff der Übernahme. Während Hegels Philosophie noch darauf abzielt, religiöse und wissenschaftliche Wahrheit miteinander in Einklang zu bringen, werden Erkenntnisse aus religiösem Glauben bei Goodman nicht mehr thematisiert. Damit entfällt von vornherein ein zentraler Punkt der Auseinandersetzung, wie sie noch von Hegel in dem Aufsatz Glauben und Wissen mit den Positionen Kants, Jakobis und Fichtes geführt worden ist. Übrig bleibt das Wissen. Und dieses wird einer grundlegenden Revision unterzogen.

Im folgenden soll zunächst darauf eingegangen werden, welche Einsichten Goodman zu diesem Schritt bewogen haben, um anschließend zu untersuchen, welche Möglichkeiten die Symboltheorie bietet, den Wissensbegriff neu zu fassen. Den Abschluß bilden Überlegungen, die nach den Neuerungen dieses Ansatzes im Kontrast zum Hegelschen Verständnis fragen.

\section{Revisionen werden notwendig}

Goodman entwickelt seine Thesen im Rahmen einer allgemeinen Symboltheorie. Diese geht davon aus, daß die verschiedenen Lebensbereiche durch unterschiedliche Symbolsysteme geprägt sind. Jedes System zeichnet sich durch charakteristische Beziehungen zwischen Zeichen aus, die sich in unterschiedlichen syntaktischen und semantischen Merkmalen äußern, sowie in der Art der Symbole. Symbole können, festgelegt durch das System, unterschiedliche Funktionen übernehmen. Zu nennen sind Denotation - die Bezugnahme eines Symbols auf einen Gegenstand - und Exemplifikation, bei der eine Eigenschaft des Gegenstandes zum Symbol wird, als die Wichtigsten, gefolgt von einer Vielzahl an Varianten. Entscheidend ist in diesem Zu- 
sammenhang die These, daß kognitive Prozesse an Symbolfunktionen gekoppelt sind. Symbolprozesse aber sind gleichzusetzen mit Erkenntnisprozessen. Dies hat zur Konsequenz, daß alle Bereiche, in denen Symbolfunktionen anzutreffen sind, von einer Theorie des Wissens berücksichtigt werden müssen.

Erkenntnistheoretische Voraussetzungen. Grundlage für Goodmans Thesen bilden erkenntnistheoretische Überlegungen. Aus der Einsicht, daß kein Zugang zum absolut Gegebenen besteht, folgert Goodman in Ways of Worldmaking, daß die Vorstellung von der einen Welt aufzugeben ist. Welt gibt es nur in einer vermittelten Form über Symbolsysteme; - die rohe, unbearbeitete Welt ist unerreichbar. Welt stellt sich nur in einer bearbeiteten Form dar, der Weise, wie die Welt ist. Die vielen korrekten Symbolsysteme, die sich konstruieren lassen, sind die Weisen der Welt. Das System verkörpert dabei eine erkenntnistheoretische Grenze. Die Frage nach einer allen Systemen zugrundeliegenden Welt ist sinnlos, da es keinen direkten Zugang gibt. Aussagen über die Welt sind immer Aussagen eingebettet in ein bestimmtes Beschreibungssystem. Es ist unmöglich, dieses hinter sich zu lassen und einen Blick auf die Welt zu werfen ohne jeden Bezugsrahmen. Die Welt ist verloren, was bleibt, sind Weisen der Beschreibung. 2

Dies ist die erste wichtige Überlegung, die zur Ablösung des traditionellen Wissensbegriffs führt: Es gibt keinen Zugang zur Welt an sich. Damit entfällt automatisch auch die Möglichkeit der Überprüfung der Wahrheit durch den Vergleich mit einer beschreibungsunabhängigen Welt. Der unbedarfte, neutrale Blick auf die Dinge bleibt verwehrt. Jedes Beobachten ist vorstrukturiert und liefert nichts als Beschreibung. Wir können nicht auf die Welt als Korrektiv zurückgreifen, um unser Wissen zu prüfen.

Zeichen jenseits der Sprache. Wichtiger noch wird aber eine weitere Voraussetzung der Symboltheorietheorie. Die zahlreichen Symbolsysteme, die Weisen der Welt, beschränken sich nicht auf sprachliche Systeme. Zeichenprozesse und damit kognitive Leistungen sind in gleicher Weise auch in außersprachlichen Bereichen anzutreffen. Entsprechend artikuliert sich Wissen nicht allein in den klassischen Wissenschaften, sondern es gibt analoge Formen in Symbolsystemen der Malerei, Musik, Architektur, des Tanzes und vielen mehr. Mit einem Mal sind Bereiche ins Blickfeld geraten, die nie zuvor für Erkenntnis als relevant erachtet wurden. Es sind Welten, die nicht allein in denotativen sprachlichen Systemen ihren Ursprung haben, sondern ebenso pikturale, perzeptive, exemplifikatorische, metaphorische und viele andere Elemente umfassen. In jeder dieser Welten vollzieht sich Erfahrung von Welt. Der Anspruch Goodmans, daß ein Musikstiuck oder ein Bild im gleichen Maße einen Verstehensfortschritt bedeuten kann wie ein physikalisches Gesetz, erschüttert die bisherige Konzeption von Wissen. Wissen ist als Fachbegriff auf verbale Systeme zugeschnitten. Einer Symboltheorie, deren Anspruch sehr viel umfassender ist, kann er nicht mehr genügen.

Ungenügende Wahrheit. Für Goodmans Projekt einer allgemeinen Symboltheorie ist auch die traditionelle Vorstellung von Wahrheit zu eng. Da sie sich auf verbale Symbolsysteme beschränkt, muß er für seinen umfassenderen Anspruch des Zeichens eine erweiterte Konzeption entwickeln. Aufgrund der erkenntnistheoretischen Voraussetzungen ist eine Überprüfung der Wahrheit entsprechend der Korrespondenzbeziehung unmöglich. So bleiben nur sogenannte sinterne Theorien, die nicht versuchen, Wahrheit durch einen Rückgriff auf die Welt festzulegen.

Im wissenschaftlichen Alltag dominieren pragmatische Überlegungen. Wer auf der Suche nach Gesetzmäßigkeiten ist, bevorzugt häufig Näherungsaussagen gegenüber der `exakteren Wahrheit‘. Beileibe nicht jede Wahrheit wird verfolgt, Trivialitäten scheiden als belanglos aus. Beobachtungen werden in einen größeren Theoriezusammenhang eingeordnet, und dadurch ist das Augenmerk auf bestimmte Aspekte gerichtet. Bei der Auswahl wissenschaftlicher Aussagen verdrängen Kriterien wie Triftigkeit, Bündigkeit, Reichweite, Informationsgehalt und Organisationskraft die Wahrheit auf einen Nebenschauplatz. Wahrheit kann dabei eine Rolle spielen, aber sie ist weder hinreichend noch notwendig. ${ }^{3}$

Gesucht ist daher eine allgemeinere Charakterisierung, die über die Sprache hinaus Symbole aller Systeme beurteilen und die Schwächen der Wahrheit ausgleichen kann. Deshalb ersetzt Goodman Wahrheit durch Richtigkeit. Im Zusammenhang mit Richtigkeit kann genauso von Bildern, Komposition, Musizierweise und Gedichten gesprochen werden wie von Schautafeln, Statistiken und allgemeinen Gesetzen. Sie kann sich aus Relevanz, Brauchbarkeit, Wirkung, Wahrheit und anderem zusammensetzen. Welche Komponenten in welcher Zusammensetzung bestimmend sind, variiert stark mit dem Kontext. Deshalb muß bei der Bestimmung der Richtigkeit immer das umgebende Symbolsystem mit in die Betrachtung einbezogen werden. Nur innerhalb eines Systems läßt sich sagen, ob etwas richtig oder unrichtig ist, »rightness [... is] relative to system ${ }^{4}{ }^{4}$ 
Kontext. Die Beobachtung, daß Symbole nicht isoliert funktionieren, sondern immer kontextgebunden sind, stellt einen weiteren, wichtigen Aspekt in der Ablösung der überlieferten Wissensvorstellungen dar. Während im traditionellen Verständnis Aussagen unabhängig für sich stehen können, macht Goodman deutlich, daß Symbolfunktionen nur innerhalb ihres komplexen Zusammenhanges verstanden werden. Erwartungen und Überzeugungen leiten unsere neuen Erfahrungen, ein implizites »Hintergrundwissen « ist an jedem Erkenntnisproze $\beta$ beteiligt. ${ }^{5}$ Nicht alleine, sondern erst gemeinsam mit einer Familie von möglichen Alternativen, kann ein Zeichen klassifizieren und begrenzen. Die syntaktischen und semantischen Eigenschaften des gesamten Systems entscheiden darüber, um welche Art von Zeichen es sich handelt. Welche Rolle das Symbol übernimmt, ob es denotiert, exemplifiziert oder ob es etwa metaphorisch gebraucht wird, definiert der Kontext. Auf diese Weise stehen sprachliche und außersprachliche Symbole in engem Zusammenhang und verweisen wechselseitig aufeinander. Die unterschiedlichen Komponenten beeinflussen sich gegenseitig in ihrer ganzen Vielschichtigkeit, so daß Wissen nicht länger allein auf Wahrnehmung gründet. Es umfaßt ebenso Abbildungen und Beschreibungen wie auch emotive und evaluative Aspekte. ${ }^{6}$

Mit dieser starken Kontextabhängigkeit und Komplexität der Symbolprozesse wird deutlich, daß Wissen nicht eindimensional in Verstand oder Vernunft anzusiedeln ist, wo klar separierte Aussagen beurteilt werden. Gesucht wird stattdessen ein Konzept, welches die multiplen Vernetzungen miteinbeziehen kann.

Prozeßhaftigkeit. Erkenntnisgewinnung ist ein dynamischer Prozeß mit offenem Ende. Zu glauben, man könnte Wissen im Fortschreiten der Wissenschaft auf sicheren Fundamenten immer weiter anhäufen, ist ein Trugschluß. Fehler sind immer möglich: »No sentences are incontrovertible, and no modes of reasoning infallible. $\aleph^{7}$ Somit unterliegt Wissen einem steten Wandel. Wie die Revisionen aussehen, ist von vielfältigen Einflußfaktoren abhängig. Bestand und Veränderung stehen hier in wechselseitiger Bezugnahme. Die tradierte Praxis greift ebenso in die Entwicklung ein wie die angestrebten Ziele oder Zwecke, die erfüllt werden sollen. Entscheidend ist, daß es sich bei diesem Prozeß um einen aktiven handelt. Das Wissen von der Welt wird nicht einfach vorgefunden, sondern es sind immer schon eigene Konstruktionsleistungen im Spiel. Der Erkenntnisprozeß wird somit auch zu einem Schaffensprozeß: „Comprehension and creation go on together. ${ }^{8}$

Keine Gewißheit. Der ständige Wandel dessen, was als Wissen gilt, macht deutlich, daß Gewißheit in diesem Zusammenhang nicht möglich ist. Gewißheit läßt sich nicht mehr halten: "Certainty - a pretentious muddle of the psychological and the pseudological - is unsalvageable. « ${ }^{9}$ Will man Gewißheit beweisen, stellt sich die Schwierigkeit, daß für die Gewißheit der Konklusion bereits die Gewißheit der Prämissen vorausgesetzt werden muß. Aber auch andere Annäherungen an den Begriff schlagen fehl. Wird Gewißheit als eine starke Überzeugung charakterisiert, dann trifft sie sowohl auf wahre als auch auf falsche Aussagen zu. Nicht besser sieht es aus, wird Gewißheit mit Wahrscheinlichkeit gleichgesetzt. Gewißheit kann also nicht helfen, wahre Aussagen von falschen zu trennen, genauso wenig dient sie dazu, Wissen näher einzugrenzen.

Gewißheit hat daher in Goodmans Philosophie keinen Platz und muß einem Konzept der vorläufigen Übernahme weichen. Ergebnisse werden übernommen und in das System eingebaut; sie können durch vielfache Bestätigung gut etabliert sein - ohne jedoch je die Sicherheit von Gewißheit zu erreichen. Sie bleiben immer Ergebnisse »unter Vorbehalt«, die nicht vor zukünftigen Veränderungen sicher sind.

\section{Verstehen als Welterzeugung}

Mit den aufgezeigten Problemen, insbesondere der Wahrheit und der Gewißheit, muß die Konzeption von Wissen insgesamt hinterfragt werden. »Knowledge«, schreibt Goodman, »involves both truth and certainty, and suffers the ailments of both along with further complications. ${ }^{10}$ Aus diesen Gründen wird eine Revision des gesamten Wissensbegriffs notwendig. Wissen wird ersetzt durch Verstehen.

Goodmans Lösungsvorschlag ist eng mit seiner Theorie der Welterzeugung verknüpft. Denn die Prozesse der Welterzeugung münden letztlich in den Vorgang des Verstehens. Ausgangspunkt sind immer bereits bestehende Welten. In Goodmans Philosophie wird Welt auch als Symbolsystem charakterisiert: ein Verband von Symbolen, der durch bestimmte syntaktische und semantische Merkmale jeweilige Besonderheiten herausbildet. Diese etablierten Symbolsysteme können nun Veränderungen erfahren. Um ein Symbol oder einen Symbolkomplex neu einzuführen, wird seine Wirkung am bestehenden System getestet. Dazu 
wird das Symbol probeweise eingefügt und versucht, es zum Passen zu bringen. Unter Passen darf man sich allerdings nicht ein passives Einpassen vorstellen; es ist ein aktiver, modifizierender Prozeß. Das Passen wird erzeugt: "The fit has to be made " ${ }^{11}$ Das bedeutet, da 3 sowohl das Symbol als auch das Umfeld Veränderungen durchlaufen, so lange, bis sie aufeinander abgestimmt sind. »The making may involve minor or major adjustments in what is being fitted into or what is being fitted in or both.« 12 Die Veränderungen des bestehenden Gefüges können dabei so stark sein, daß es einer Revision des gesamten Symbolsystems gleichkommt.

Doch nicht immer läßt sich ein Symbol einpassen. Die Kohärenz des Systems muß weiterhin gewährleistet sein. Ein Symbol, das diese Anforderung verletzt, kann nicht dauerhaft übernommen werden. Neuerungen kann sich auch der vorhandene Symbolverband widersetzen. Letzterer bildet ein Hintergrundgefüge, welches, je länger es in Kraft ist, sich desto stärker einer Revision entgegensetzt. Sein Beharrungsvermögen ermöglicht eine gewisse Konstanz und damit letztlich die Ausbildung von Symbolsystemen. Das heißt aber nicht, daß Innovation damit unmöglich wird. Gewohnheiten können sich zwar Veränderungen entgegenstellen und eine Übernahme verhindern, oft genug gelingt aber die Akzeptanz eines Symbols. All die alteingesessenen Übernahmen geben dann dem neuen Anwärter die Richtung vor. Mit der Dauer der Verankerung wächst auch sein Anspruch auf Richtigkeit. Jede erfolgreiche Übernahme hat Auswirkungen auf den Hintergrund und trägt zu dessen Veränderung bei, wenn auch nur als schleichender Prozeß. Im Laufe der Zeit kann es auf diese Weise durchaus zu einem Wandel etablierter Praktiken und Prinzipien kommen.

Wurde ein Symbol erfolgreich eingepaßt, zeigt sich das an seinem Wirken. Von Interesse ist dabei weniger das Wirken des Symbols als das Wirken des gesamten Gefüges. Lassen sich mit der Übernahme neue Einsichten gewinnen, werden Probleme plötzlich lösbar oder ergeben sich gewünschte Anwendungen, dann hat das Symbol gute Arbeit geleistet. Können aufschlußreiche Verbindungen hergestellt, signifikante Unterscheidungen entdeckt oder Anomalien beseitigt werden, ist das auf veränderte Symbolsysteme zurückzuführen. Diese Weiterentwicklungen bewirken dann ein tieferes Begreifen und Verstehen.

Das Wirken kann somit als Test für das Passen dienen: hat die Übernahme eines Symbols die geschilderten Konsequenzen zur Folge, dann paßt es in das System. Was allerdings die gewünschten Konsequenzen sind, kann sich im Laufe der Zeit ändern. »Even what constitutes fitting and working may undergo change, may itself have to be adjusted in order to fit and work. ${ }^{13}$

Wissen verstehen. Wissen, als Akkumulation wahrer Aussagen, über die Gewißheit herrscht, wird von einem dynamischen Prozeß abgelöst, dem Verstehen. Statt von Wahrheiten Sicherheit zu erlangen, werden neue Symbole probeweise übernommen. Wenn es gelingt, die Übernahme dauerhaft im System zu plazieren, steigt auch mit zunehmender Verankerung der Anspruch auf Richtigkeit. Das System wird auf diese Weise erweitert und falls erforderlich, in einem wechselseitigen Prozeß modifiziert. Kann das Symbol zum Wirken gebracht werden, dann ist ein neues, tieferes Verstehen erreicht. Verstehensfortschritt ist die Folge, ein Prozeß, der immer weiter geführt werden muß. »The process goes on and on, for understanding is always partial; fitting symbols or ways of symbolizing in and making them work is a task as varied as are symbol systems, referential relationships, and situations and objectives. ${ }^{14}$ Erkenntniszuwachs wird gleichgesetzt mit einem Fortschritt des Verstehens, der Verbesserung von Fertigkeiten, in Vertiefung oder Verfeinerung des Vorhandenen.

Was hier beschrieben wurde, sind Prozesse der Welterzeugung. Welterzeugung ist ein aktiver Vorgang. Neue Strukturen werden entworfen, neue Ordnungen werden ausprobiert. Es sind diese Prozesse, die das Verstehen bestimmen. »All the processes of worldmaking I have discussed enter into knowing. [...] Comprehension and creation go on together. ${ }_{15}$ Verstehen darf nicht als rezeptives Aufsammeln von Wahrheiten verstanden werden, sondern muß ständig neu erarbeitet werden. »If worlds are as much made as found, so also knowing is as much remaking as reporting. "16 Im Welterzeugen schreitet das Verstehen fort. Indem Systeme zum Passen und Wirken gebracht werden, werden Welten erschaffen und gleichzeitig ein Beitrag zum Verstehen geleistet. Welterzeugung und damit Verstehen sind kein statischer Zustand, sondern ein dynamischer Prozeß. Ein Prozeß, der Aktivität voraussetzt und keinen Wandel ausschließt. 


\section{Goodman nach Hegel}

Wirft man von Goodman aus einen Blick zurück auf Hegel, stellt sich die Frage, was sich mit der Neukonzeption geändert hat. Bereits Hegel zielt mit seiner Schrift Glauben und Wissen durch seine Kritik an Kant, Jakobi und Fichte auf einen veränderten Wissensbegriff. Aber er kann sich letztlich in seiner Philosophie nicht den traditionellen Wissensvorstellungen entziehen - sie bleibt einem kumulativen Wissensbegriff verhaftet, der auf Gewißheit und Wahrheit der zugrundeliegenden Aussagen aufbaut. Der eigentliche Bruch mit den traditionellen Vorstellungen kündigt sich erst im Laufe des 20. Jahrhunderts an und findet im Werk Nelson Goodmans eine exemplarische Ausformulierung.

Dennoch finden sich rückwirkend betrachtet bereits bei Hegel Elemente, die für eine spätere Konzeption von Wissen als Verstehen von Bedeutung werden. Den Ansätzen Goodmans und Hegels ist gemeinsam, daß beide nicht auf eine unabhängig vom Subjekt gegebene Welt zurückgreifen können: hier die Welt als Ergebnis von Konstruktionsleistungen, dort die Beschränkung auf das Subjekt und dessen Bewußtsein. Welt wird bei Hegel nicht mehr als Ding gesehen, ohne daß er gleichzeitig in den »alten Dogmatismus« der Reflexionsphilosophie zurückfällt, der die Welt auf ein "System von Erscheinungen, oder von Affectionen des Subjects, und geglaubten Wirklichkeiten « beschränkt. ${ }^{17}$ Damit entfällt die intuitiv einfache Darstellung von Wissen: Wissen als wahre Aussagen, wahre Aussagen als solche, die einen bestehenden Sachverhalt in Übereinstimmung mit der Welt ausdrücken. Gemeinsamkeiten treten auch bei der Lösung des Problems auf: Wissen beziehungsweise Verstehen wird durch eine innere Dynamik etabliert. Was bei Goodman von der vorläufigen Übernahme geleistet wird, welcher der Prozeß des Passens und Wirkens folgt, übernimmt bei Hegel die dialektische Grundfigur. Obwohl erst in späteren Schriften ausgearbeitet, findet sich dieser Ablauf bereits in Glauben und Wissen. Mit der Diskussion der drei philosophischen Positionen zeigt Hegel, daß hier die "Metaphysik der Subjectivität [...] den vollständigen Cyclus ihrer Formen in der Kantischen, Jakobischen und Fichte'schen Philosophie durchlaufen« hat. ${ }^{18}$

Doch an diesem Punkt enden die Gemeinsamkeiten. Während die Entwicklung bei Hegel von vornherein ein festgelegtes Ziel hat, wird Verstehen bei Goodman zu einem offenen Prozeß, der niemals abgeschlossen ist. Er ist ein aktiver, dynamischer Vorgang, er resultiert aus Probieren, Konstruieren und Testen. Ziele und Zwecke im Verstehensprozeß sind niemals endgültig festgelegt, sie können sich wandeln, abhängig von dem, was erreicht werden soll. Verstehen selbst kann sich so im Laufe der Zeit einer Entwicklung unterziehen und auf historische Veränderungen reagieren, wohingegen der Hegelsche Weg ein Weg zum absoluten Wissen ist, mit einem eindeutigen Endpunkt.

Während Wissen bei Hegel allein eine Angelegenheit der Vernunft ist, wird Verstehen durch eine große Komplexität geprägt, es ist eingebunden in die Zusammenhänge von Handlung, Werten und Emotionen. Entscheidend ist die große Bandbreite an Bereichen, in denen sich Verstehen abspielt, neben den sprachlichen auch eine Fülle von außersprachlichen. Alle Systeme teilen bestimmte Zeichenfunktionen. Folglich unterscheiden sich Künste und Wissenschaften, ebenso wie Natur- und Geisteswissenschaften nicht grundsätzlich, sondern nur graduell.

Ziele und Zwecke im Verstehensprozeß müssen immer wieder geprüft werden. Verstehen entgeht damit einem fragwürdigen Dogmatismus: Ein nicht hinterfragbares und nicht wandelbares Endziel des Wissens wird verworfen. Ziele und Zwecke sind dabei nichts weiter als temporäre Festsetzungen - Festsetzungen, die sich ständig neu bewähren müssen. In diesem fortwährenden Infragestellen liegt ein aufklärerisches Element. Indirekt wird so der Welterzeuger mit in die Pflicht genommen: Verantwortung und kritische Prüfung sind gefragt. Aktiv erzeugte Verstehensprozesse konstituieren die jeweilige Welt. Jeder am Verstehensprozeß Beteiligte ist auch daran beteiligt, Welt zu schaffen. Aufgeräumt wird auf diese Weise auch mit dem Klischee vom unschuldigen, unbedarften Grundlagenforscher: wer versucht, neue Bereiche zu erschließen, gestaltet Welt aktiv und trägt damit letztlich auch Verantwortung dafür, wie sie sich entwickelt. 


\section{ANMERKUNGEN}

1 G.W.F. HEGEL, »Glauben und Wissen«, in: Gesammelte Werke, Bd. 4, Hamburg 1968.

2 N. GOODMAN, Ways of Worldmaking, Indianapolis 1978, dt.: Weisen der Welterzeugung, Frankfurt/M. $1984,4$.

3 Ebd., Kap. 1.5/7.3.

4 Ebd., 139.

5 N. GOODMAN / C.Z. ELGIN, Reconceptions in Philosophy and Other Arts and Sciences, Indianapolis 1988, dt.: Revisionen: Philosophie und andere Künste und Wissenschaften, Frankfurt/M. 1989, 5.

6 Ebd., 4.

7 Ebd.

8 GOODMAN, Ways of Worldmaking, 22.

9 GOODMAN / ELGIN, Reconceptions, 159.

10 Ebd., 155.

11 Ebd., 158.

12 Ebd.

13 Ebd., 159.

14 Ebd., $161 \mathrm{f}$.

15 GOODMAN, Ways of Worldmaking, 22.

16 Ebd.

17 HEGEL, „Glauben und Wissen«, 412.

18 Ebd. 